\title{
Alexander Kluge/Gerhard Richter. L'art contre le hasard
}

Alexander Kluge/Gerhard Richter. Die Kunst gegen den Zufall

Alexander Kluge/Gerhard Richter. Art against chance

Maud Hagelstein et Céline Letawe

\section{(2) OpenEdition}

\section{Journals}

Édition électronique

URL : http://journals.openedition.org/ceg/1143

DOI : $10.4000 /$ ceg. 1143

ISSN : 2605-8359

Éditeur

Presses Universitaires de Provence

Édition imprimée

Date de publication : 18 décembre 2015

Pagination : 105-116

ISBN : 979-1-03200-020-5

ISSN : 0751-4239

\section{Référence électronique}

Maud Hagelstein et Céline Letawe, «Alexander Kluge/Gerhard Richter. L'art contre le hasard », Cahiers d'Études Germaniques [En ligne], 69 | 2015, mis en ligne le 17 décembre 2017, consulté le 26 novembre 2020. URL : http://journals.openedition.org/ceg/1143 ; DOI : https://doi.org/10.4000/ceg.1143 


\section{Alexander Kluge/Gerhard Richter. L'art contre le hasard}

Maud HAGELSTEIN

Université de Liège

Céline LETAWE

Université de Liège

Dans plusieurs de ses textes, Alexander Kluge affronte le problème du temps, qui s'écoule sans discontinuer, avec des effets tantôt heureux tantôt désastreux. La formulation de ce problème implique presque toujours la référence au cours hasardeux des choses. Quel hasard fait qu'entre plusieurs indéterminations - c'est-àdire plusieurs virtualités encore susceptibles d'êtres actualisées - un seul événement est soudainement fixé de manière irréversible? La détermination hasardeuse et brutale du cours des choses révèle son caractère potentiellement injuste: pourquoi le monde prend-il telle tournure (éventuellement catastrophique) plutôt que telle autre? Pourquoi certains sont-ils épargnés quand d'autres ne le sont pas? Confronté à ce problème insistant, Alexander Kluge nous donne les moyens - par ses réflexions théoriques mais aussi par le dispositif pratique mis au point dans ses œuvres - de repenser la fonction de l'art: les œuvres, visuelles ou littéraires, pourraient avoir pour vocation de dompter le flux fatal du temps, de suspendre l'« emportance » du temps - pour citer une traduction d'Hilda Inderwildi et de Vincent Pauval ${ }^{1}$-, en mettant «sous tension» nos représentations du monde et en y réintroduisant des indéterminations pour nous inviter à repenser l'histoire aussi bien dans sa terrible détermination que dans ses alternatives. Le travail entamé avec l'artiste allemand Gerhard Richter nous semble pouvoir être lu dans cette perspective.

En 2010, Alexander Kluge et Gerhard Richter publient un premier livre commun sous le titre Décembre: 39 histoires rédigées par Alexander Kluge, assorties de 39 clichés photographiques pris par Gerhard Richter ${ }^{2}$. A priori, l'iconographie n'est pas complexe. Tous les clichés montrent la même chose, à savoir les sapins enneigés des Grisons, dans le sud-est de la Suisse, qui dominent les fameuses montagnes de Sils-Maria où est né le projet d'un livre commun. Dans ces mêmes montagnes, un siècle plus tôt (1881), Nietzsche eut la révélation de l'" éternel retour » - élément anecdotique mais non anodin puisque Décembre se présente, au premier regard, comme une méditation sur le temps. La quatrième de couverture de la traduction

Alexander Kluge, Gerhard Richter, Décembre, trad. de l'all. par Hilda Inderwildi et Vincent Pauval, Zürich-Biel/Bienne-Berlin, diaphanes, 2012, p. 74.

2 Alexander Kluge, Gerhard Richter, Dezember - 39 Geschichten, 39 Bilder, Frankfurt am Main, Suhrkamp, 2010. 
française indique d'ailleurs qu'Alexander Kluge y compose son «almanach du siècle ». Les 39 images manifestent d'abord une grande monotonie; certaines sont presque identiques ${ }^{3}$. On y voit partout le même sujet: des arbres recouverts de neige. Seul le cadrage varie; on évolue de plans très rapprochés à des vues plus larges sur la vallée. On ne voit jamais le ciel, même si certains plans panoramiques suggèrent un horizon que la brume nous cache ${ }^{4}$.

Les 39 histoires d'Alexander Kluge - qui ont souvent la brièveté d'informations journalistiques - viennent se cogner à ces vignettes, casser leur monotonie et rompre l'uniformité de l'ensemble. L'agencement dans lequel les 39 images et les 39 textes sont pris aurait probablement pu être différent, même s'il n'est pas tout à fait aléatoire, puisque les histoires sont classées non pas chronologiquement (on va de 1991 à 1941 en passant par l'an 21999 avant Jésus-Christ) mais selon l'ordre des jours du mois de décembre. Nous avons ici choisi de proposer une lecture, à partir du thème du hasard, qui nous semblait prégnant. Comme évoqué en introduction, il y a bien une question qui se pose avec insistance tout au long de Décembre, mais pas seulement: comment se fait-il qu'entre une multiplicité de possibles, un événement survienne (ou ne survienne pas) qui peut (ou aurait pu) tout changer et faire basculer de manière irréversible notre histoire? On parlera donc ici des éléments de hasard qui empêchent la maîtrise totale, par l'homme, de son destin ${ }^{5}$.

Les brèves rapportées dans Décembre évoquent parfois la rubrique "faits divers » d'un quotidien - celle qu'on appelle plus familièrement « rubrique des chiens écrasés ». Alexander Kluge s'inspire de cette formule journalistique qui consiste à rassembler l'inclassable - c'est-à-dire à classer ensemble les événements qui ne correspondent à aucune catégorie de base d'un média d'actualité. La rubrique « faits divers » reprend généralement sans les lier entre elles les nouvelles qui ne relèvent ni de l'international, ni du national, ni de la politique, ni de l'économie, ni de la culture, etc. Selon l'usage commun, il s'agit d'événements tragiques, crimes, vols et accidents, dont la portée paraît souvent secondaire au regard des grands faits d'actualité. Parmi les couches dites cultivées, on trouvera toujours quelqu'un pour critiquer les médias qui accordent à ces faits divers une importance démesurée: une certaine presse reposerait entièrement sur l'attirance des couches populaires pour ce genre d'événements tragiques.

3 Voir par exemple Kluge, Richter, Décembre, p. 85 et 91.

4 Voir la dernière image (ibid., p. 126). Cette œuvre bicéphale fonctionne comme un vaste système d'échos. On remarquera une première correspondance entre le programme iconographique établi par Richter pour ce projet et l'axe thématique autour duquel tournent un certain nombre de textes: le hasard, Zufall en allemand, fait référence à ce qui tombe, plus précisément à ce qui nous tombe dessus (was uns zufällt). La neige - qui constitue le sujet principal des images de Gerhard Richter - peut donc répondre visuellement à ce problème. Certes, elle apparaît parfois dans toute sa légèreté (comme une poussière dans l'air, une fumée ou un voile très fin), mais elle se manifeste surtout dans toute sa lourdeur, pesant sur les branches, et on en fait l'épreuve visuelle: la neige qui s'entasse menace à chaque instant de tomber.

5 Phénomène qui n'est pas sans générer quelque résistance, notamment dans le champ scientifique: Alexander Kluge décrit les multiples tentatives - souvent vaines - par lesquelles l'homme tente de contrôler/comprendre le temps (prédictions météorologiques, calendriers, théorie évolutionniste, norme industrielle allemande DIN, etc.). 
Alexander Kluge propose ici une série d'historiettes dont la tonalité dramatique est renforcée ou atténuée, selon les cas, par les photographies. Formellement et thématiquement, ces brèves inclassables s'inscrivent dans le registre du fait divers et s'en extraient en même temps par l'effet de leur mise en contexte: Kluge renverse l'idée commune selon laquelle les faits divers sont généralement secondaires puisque, dans ses récits, les accidents et autres coups du hasard ont un impact indéniable sur le devenir de l'humanité. C'est ce qu'on appelle communément « l'effet papillon ».

On peut reprendre pour commencer l'histoire qui sert de parabole à ce thème du hasard ${ }^{6}$. Kluge y raconte un accident tout juste évité, dans un climat de pluie verglaçante - on note d'ailleurs que les intempéries et autres caprices météorologiques affectent dans Décembre les principaux récits d'accident. Dans la soirée du 3 décembre 1931, en repartant de la noce des époux Goebbels, les chauffeurs respectifs d'Hitler, témoin du mariage, et de la mariée elle-même, évitent de justesse la collision fatale. Le chauffeur de la Maybach rouge de la nouvelle Madame Goebbels tentait de dépasser dans un long virage la Mercedes noire d'Hitler. Kluge: «En cette époque d'avènement de l'automobilisme, il n'était pas parvenu à la connaissance de grand monde qu'il est contre-indiqué de freiner sur une surface verglacée et de conduire avec un coup dans le nez ${ }^{7} \gg$. Le texte se poursuit par un commentaire dialogué entre deux personnes (dont on peut supposer qu'il s'agit de Kluge et Richter eux-mêmes) : " -C'est à la seule providence que les deux véhicules durent de s'éviter. - Qu'entendez-vous par providence ${ }^{8}$ ? $\gg$ Dans une interview de 2010, à la question de savoir si c'est là le fait de la providence, Kluge répond que c'est l'action du diable ${ }^{9}$. Dans Décembre, par contre, la question reste - à cet endroit du moins - sans réponse. Le texte se termine par une note de bas de page, dans laquelle Kluge démontre son lien personnel au fait divers: «Logé dans l'abri bien tempéré du ventre, je faillis naître sans qu'Hitler ait pris part à l'avenir. Il manqua $40 \mathrm{~cm}$ pour qu'eût lieu une collision mortelle entre les grosses cylindrées sur la piste verglacée ${ }^{10} »$. Protégé du froid glacial du mois de décembre, Kluge, dans le ventre « bien tempéré » de sa mère, devait encore attendre un peu plus de 2 mois pour naître (il est né le 14 février 1932). Selon les standards, un bébé à 7 mois de gestation mesure environ $40 \mathrm{~cm}$.

En résonance avec ce récit, Kluge reprend et décrit dans Décembre plusieurs autres « accidents » liés au hasard (en particulier au « hasard météorologique ») : un accident de voiture causé par le verglas le 23 décembre $1999^{11}$; un viaduc ferroviaire emporté par le courant d'un fleuve gonflé par des pluies inhabituelles au moment

6 Ibid., p. 10-11.

Ibid.

8 Ibid. L'insertion de dialogues dans les récits est un processus récurrent chez Kluge, qui signale par ailleurs que l'incident fit beaucoup parler. Il est néanmoins tentant de supposer ici que l'échange a lieu entre les deux artistes.

9 « Der Teufel hat die Hand dazwischen gehalten », in Philip Holstein, " Gerhard Richter zeigt den Winter », Rheinische Post, 21.10.2010, disponible en ligne: http://www.kluge-alexander.de/literarischerautor/selbststaendige-werke/detailansicht/artikel/gerhard-richter-zeigt-den-winter.html.

${ }^{10}$ Kluge, Richter, Décembre, p. 11.

11 Ibid., p. 73. 
même du passage d'un train bondé le 12 décembre $2009^{12}$, etc. Mais le hasard, c'est aussi, comme du reste dans le récit impliquant Hitler, l'accident qui ne survient pas. Le 24 décembre 1943 au soir, un médecin pratique un accouchement délicat alors qu'il a consommé quatre verres de schnaps, ce qui a causé « l'inertie de pas mal de ses nerfs ${ }^{13} »$. Contrairement à ce que semble d'abord suggérer la forme du récit, l'issue est heureuse.

Un autre récit important semble répondre à celui de l'accident de voiture manqué. Le 8 décembre 1941, annoncé comme un « lundi chômé ${ }^{14}$ », le Führer sort de son lit à 11 heures. Pour une raison qui n'est pas explicitée, la rencontre prévue avec toutes les instances concernées par l'avenir du Reich n'a pas lieu et se voit finalement reportée au 20 janvier 1942. Cette réunion sera connue sous le nom de « conférence de Wannsee »- conférence pendant laquelle quinze hauts responsables du Reich décidèrent de l'organisation logistique (administrative, économique et technique) de l'extermination du peuple juif. Kluge se demande si la conférence se serait déroulée autrement un mois et demi plus tôt, à la date initialement prévue. En raison de l'urgence, les décisions n'auraient probablement pas été les mêmes. Kluge fait dans ce cadre référence au darwiniste Horst Boecker, travaillant à une « Histoire des espèces du mal »: «Comment, telle est sa question, un ensemble confus d'indéterminations finit-il par produire UNE DÉTERMINATION particulière qui mène au désastre, tandis que d'autres indéterminations ("potentialités") simplement se dissipent ${ }^{15}$ ».

Ces deux récits présentent donc deux moments de hasard (un accident évité et une réunion déplacée) qui auraient pu être autrement déterminés et n'avoir pas de telles conséquences sur l'histoire allemande. Plus largement, l'auteur de ces récits envisage l'influence que de tels moments peuvent avoir sur l'histoire de l'humanité. En nous poussant par exemple à réfléchir aux possibilités qui auraient pu rejouer autrement le premier meurtre de l'histoire chrétienne, il montre que l'évolution de l'humanité ellemême ne tient qu'à un fil: "Qu'advient-il si Caïn, brandissant la pierre, est retenu par quelque aléa ou ange, si p. ex. il dégringole une pente, laisse échapper sa pierre, trouve la mort, et qu'Abel [...] devient l'aïeul exclusif du genre humain ${ }^{16}$ ?».

À travers ses histoires d'accidents effectifs ou avortés, Kluge construit une vision du monde où les indéterminations se transforment brutalement selon un ordre hasardeux en configurations événementielles déterminées. Tout se joue finalement à quelques instants près, à quelques mètres ou centimètres près. C'est également ce qui ressort du texte daté du 23 décembre $1999^{17}$. L'auteur d'un traité à paraître sur le temps, Fred Kelpe, pris dans une tempête de verglas, subit un sévère accident de la route et doit passer plusieurs semaines à l'hôpital. Kelpe travaille à une théorie très complexe et profite de sa convalescence forcée pour avancer sur son manuscrit. Selon cette théorie, le temps serait un monstre impétueux emportant tout sur son passage. C'est Chronos, le temps qui fauche et qui engloutit; il « fonce tête baissée

\footnotetext{
${ }^{12}$ Ibid., p. 41.

13 Ibid., p. 77-78.

14 Ibid., p. 26.

15 Ibid.

16 Ibid., p. 94.

${ }_{17}$ Ibid., p. 73-75.
} 
vers l'avenir ». « Le temps qui emporte [reißende Zeit], dit Kelpe, est l'unique forme du destin que vous puissiez sentir passer avant que le coup porte ${ }^{18} »$. Cela entraîne une injustice fondamentale, celle d'un processus tempétueux et sélectif, comme l'exprime la phrase suivante: «À quelques lieues de là à peine, à quelques instemps près, le temps demeure sans emportance [nicht-reißende Zeit]. Un individu à cinq mètres de distance d'une dépression de temps [Zeitabriß] est sauvé ${ }^{19}$ ».

Une fois posé ce constat - finalement banal sur le fond (peut-être moins sur la forme, allusive et apparemment anecdotique, choisie par les auteurs) -, reste à savoir ce que l'action artistique et/ou politique peut changer à la situation. Aux effets de Chronos, il convient d'opposer l'usage stratégique du Kairos - le temps de l'instant, de l'occasion opportune et de la circonstance. Le temps de l'action humaine efficace. Kluge, qui a créé en 1963 la société de production Kairos-Film, ne se contente évidemment pas de décrire l'homme en victime de l'emportance du temps. Mais il esquisse pour l'artiste des voies d'issue, notamment dans un texte plus récent sur lequel notre analyse va se concentrer à présent.

On retrouve en effet la thématique du hasard qui change le cours de l'histoire, petite ou grande, dans le deuxième livre commun de Kluge et Richter: Nachricht von ruhigen Momenten ${ }^{20}$, littéralement « Nouvelle de moments calmes ». Plusieurs histoires brèves rejouent des scénarios similaires, à nouveau empruntés au registre des accidents manqués. Dans un texte intitulé " glücklicher Zufall ${ }^{21}$ » (" heureux hasard »), déjà inclus dans Chronique des sentiments, un enfant de cinq ans glisse d'un ponton; sa tête passe à un millimètre de la structure en métal, grâce à un mouvement qu'il fait par hasard. Un peu plus tôt dans le livre, après avoir raté leur avion, un groupe d'enfants embarque dans un autre avion, qui s'écrase. Les assistantes d'un fonctionnaire avaient empêché les deux enfants de leur patron de monter à bord, juste avant le décollage. Conséquence d'un certain «Zufalls-Sinn ${ }^{22}$ », d'un «sens du hasard », d'une intuition de ces deux assistantes?

Kluge donne dans ce texte une définition du hasard singulière (voir supra), qu'il attribue au philosophe Rudolf Steiner :

Was ist Zufall? Das, was mir zufällt. Die Großzügigkeit von Natur und Umständen bringt mir einen Zugewinn. Das Gegenteil wäre Abfall. ${ }^{23}$

On note ici un jeu intéressant sur les composés allemands Zufall, hasard, et Abfall, déchet, qui sont explicitement opposés l'un à l'autre. D'une part l'événement qui «me tombe dessus » par hasard, c'est-à-dire l'événement qui, parmi tous les possibles, se réalise, et d'autre part toutes les possibilités non réalisées, finalement perdues, et donc assimilées ici à des déchets ou scories. On peut penser à cet égard au projet d'atlas établi par Richter - vaste collection de photographies, dessins et

18 Ibid., p. 74.

19 Ibid.

${ }^{20}$ Alexander Kluge, Gerhard Richter, Nachricht von ruhigen Momenten : 89 Geschichten, 64 Bilder, Frankfurt am Main, Suhrkamp, 2013. Étant donné qu'il n'existe pas encore de version française de ce livre, toutes les traductions françaises proposées ici sont les nôtres.

${ }^{21}$ Ibid., p. 30.

22 Ibid., p. 20.

23 Ibid. 
coupures de presse rassemblée à partir des années 1960. L'artiste considère les matériaux visuels rassemblés comme le fonds documentaire ou l'imagier sur lequel s'appuie sa création. Réserve d'images qui ont le statut d'esquisses. Certaines se « réalisent» et trouvent la forme d'une œuvre d'art, d'autres non. L'atlas peut donc s'envisager comme un répertoire de "déchets" (Abfall) ou un inventaire des «scories», conservés dans des registres ${ }^{24}$, repris sur le site de l'artiste ${ }^{25}$ et régulièrement exposés ${ }^{26}$.

Le hasard opère dans un instant très bref - plusieurs histoires illustrent l'idée, déjà avancée dans Décembre, qu'à quelques secondes près, les choses auraient pu être totalement différentes. L'instant où tout se joue semble particulièrement fasciner Kluge. Dans l'interview filmée qu'il réalise de Gerhard Richter en octobre 2012 ${ }^{27}$, Kluge revient sur une photo qui n'a finalement pas trouvé de place dans le livre, en dépit du fait qu'elle lui ait inspiré le tout dernier texte: l'image montre une femme qui s'apprête à poser la main sur une balustrade pour garder l'équilibre, pour ne pas tomber. Le livre se termine par ces mots : « Eine Fotografie hält den Moment fest, bevor die Hand das Geländer fasst. HANDFESTER HALT: WIRKLICHKEIT ${ }^{28}$ ", littéralement: "Une photographie fixe le moment qui précède celui où la main saisit la balustrade. APPUI SOLIDE: RÉALITÉ ». Mais « Halt» ne signifie pas seulement l'appui, la prise, et l'équilibre qui en résulte. " Halt» signifie également arrêt. La réalité, ce serait donc à la fois le contact entre la main et la balustrade, contact qui suit le moment fixé par la photographie, et l'instant même arrêté par la photographie (une photo avec un très bref temps d'ouverture du diaphragme se dit aussi «Momentaufnahme » en allemand, enregistrement de l'instant - mot qui résonne avec le terme français instantané). Il y a une très forte tension dans cet instant où tout peut arriver, et cette tension retient Kluge. Il semble littéralement fasciné par l'intuition du photographe qui déclenche ici l'obturateur juste au bon moment (« die Ahnung des Fotografen für den richtigen Moment »).

Partant de cette photographie restée invisible dans le produit final, on interrogera une première fois la capacité spécifique de l'artiste à résister à l'emportance du temps - à sa fulgurance mais aussi à son unidimensionnalité, c'est-à-dire au fait qu'un événement écrase pour ainsi dire au moment de son institution toute une série de possibles qui n'auront jamais lieu. Quand le photographe saisit « au vol » la main qui cherche à s'agripper, il capte ce qui précède la réalité (et qui en même temps ne peut être autre chose qu'elle), à savoir: l'instant presque suspendu où rien n'est encore arrivé et où plusieurs issues sont possibles. Que ce soit à travers les mots ou en utilisant les images, l'artiste aurait ce pouvoir spécifique de fixer pour un instant (même et surtout furtif) la course des choses.

Ce n'est pas pour rien que Kluge et Richter, dans leur livre sur les « moments

${ }^{24}$ Gerhard Richter, Atlas, éd. par Helmut Friedel, New York City, D.A.P./Distributed Art Publishers, 2007.

$25 \mathrm{https}: / /$ www.gerhard-richter.com/fr/art/atlas.

${ }^{26}$ On mentionnera entre autres l'exposition « Gerhard Richter : Atlas - Mikromega » qui a eu lieu du 23 octobre 2013 au 9 février 2014 au Kunstbau München.

27 Alexander Kluge, Gerhard Richter: Bildermacher, dctp, 18.03.2013 (interview filmée).

${ }^{28}$ Kluge, Richter, Nachricht von ruhigen Momenten, p. 136. 
calmes », évoquent la définition benjaminienne de l'artiste comme "grand orchestrateur du hasard»: Walter Benjamin aurait utilisé ce concept de « Zirkusdirektor des Zufalls » pour désigner l'un des membres du Bauhaus. Le texte écrit par Kluge à partir de cette proposition précise de manière particulièrement intéressante le lien entre vocation artistique et hasard, et la tension est un élément constitutif de ce lien:

\begin{abstract}
Walter Benjamin nannte ein ihm bekanntes Mitglied des Bauhauses einen « ZIRKUSDIREKTOR DES ZUFALLS ». Er verstand die Bemerkung als Ermunterung. Es sei nämlich schwer, sagte er, DIESE WIDERSPENSTIGE, AUF ÜBERRASCHUNG ZIELENDE RASSE WIRKLICHER VERHÄLTNISSE, DIE WIR ZUFALL NENNEN, die sich fast nie auf der Durchschnittstrecke bewege, aus der sich aber Geschick und die Umstände komponieren, publikumsgerecht für den Zirkus zu dressieren. Vollendet sei diese Kunst dann, wenn die Zufälle sich noch ganz in ihrem wilden oder halbwilden Zustand befänden und doch dem Gesetz der Form, das unter der Zirkuskuppel gilt, sich einfügen. Die Spannung, sagt Walter Benjamin, zwischen UNBEHERRSCHBARKEIT und DISZIPLIN, zwischen chaotischer Basis (auf diesem Boden würden die Artisten zerschellen) und dem Trapez oben: Das ist das Prinzip der Kunst. ${ }^{29}$
\end{abstract}

Il ne faut pas se méprendre: l'idée avancée ici n'est pas que l'artiste aurait la capacité d'infléchir le hasard. Mais bien qu'il peut, par son art, fixer un instant de tension, un instant autrement fuyant, où une chose ne s'est pas encore jouée, où le hasard n'a pas encore frappé - c'est-à-dire, pour évoquer un autre jeu sémantique prégnant chez Kluge, un moment où le dessein (« Wille ») n’est pas encore déterminé. L'artiste serait capable de dresser/dompter la sauvagerie du temps hasardeux, ou de brider son emportement fougueux. Le hasard n'a évidemment pas de valeur positive ou négative en soi et Kluge préserve d'ailleurs généralement ses analyses du duel axiologique entre bien et mal. Le hasard accompagne les moments où les choses se fixent, que l'issue soit heureuse ou désastreuse. Mais l'artiste peut produire une forme à partir de là, une forme qui serait en quelque sorte pleine de virtualités, une forme qui ferait voir de quelle tension ou de quel déséquilibre elle est l'image fixée et disciplinée. Aux représentations linéaires/univoques du monde, l'artiste, qu'il soit écrivain, cinéaste ou photographe, oppose des images en tension. L'image photographique, en particulier, semble avoir aux yeux des auteurs ce pouvoir très singulier - elle joue en tout cas dans cet ouvrage un rôle central, et pas seulement en tant que moyen d'expression privilégié.

Un autre exemple renforce cette idée. Sur l'image photographique (cette fois reprise dans le livre), Richter est parvenu à fixer un autre moment tout à fait hasardeux, à lui donner une forme: le modèle d'une ville du futur apparaît dans la

${ }^{29}$ Ibid., p. 31. « Walter Benjamin a qualifié une de ses connaissances membre du Bauhaus de "GRAND ORCHESTRATEUR DU HASARD”. Une remarque qu'il comprenait comme un encouragement. En effet, disait-il, il est difficile de dompter pour le cirque CETTE RACE DE SITUATIONS RÉELLES, RÉCALCITRANTE ET VISANT LA SURPRISE, QUE NOUS APPELONS HASARD, qui ne se meut presque jamais sur la trajectoire moyenne dont se composent pourtant les destinées et les circonstances. Cet art est accompli quand les hasards se trouvent encore dans leur état sauvage ou semi-sauvage mais s'adaptent néanmoins à la loi de la forme qui prévaut sous le chapiteau. La tension, dit Walter Benjamin, entre EMPORTEMENT et DISCIPLINE, entre la base chaotique (les artistes se fracasseraient sur ce sol) et le trapèze là haut: tel est le principe de l'art. » 
coupe d'un arbre ${ }^{30}$. Une partie du tronc n'a pas été coupée nettement mais semble comme arrachée, et la fibre du bois suggère un ensemble miniature de gratte-ciels: «Le fait que les gardes forestiers se soient arrêtés justement à ce moment et que cette structure soit apparue relève du hasard ${ }^{31} »$. Un hasard fixé par le photographe. Et donc, une photographie issue d'un double hasard. Richter assume cette méthode et insiste lui-même, notamment dans l'interview qu'il accorde à Kluge en octobre 2012, sur le rôle du hasard dans sa pratique photographique. Il se définit comme un homme aux aguets, toujours à la recherche de l'image intéressante. Parfois, par hasard (« zufällig »), il est le témoin d'un moment privilégié et prend alors une photo.

Comme on le voit avec l'exemple que l'on vient d'évoquer, de telles images ont le pouvoir de faire tenir ensemble - quitte à les défaire aussitôt - des choses apparemment hétérogènes (la forêt, la ville) et de produire inévitablement du sens (l'idée forcée, par exemple, que là où on coupe un arbre, une mégapole est en gestation). De manière générale, les recherches communes de Kluge et Richter, ainsi qu'une partie de leurs recherches personnelles, se donnent pour défi de rassembler l'inclassable, c'est-à-dire de faire tenir ensemble, dans une forme ouverte et non définitive, des éléments apparemment hétérogènes. Ils nous invitent de la sorte à ouvrir nos représentations habituelles à des significations nouvelles.

La presse n'intervient pas par hasard dans la rencontre des deux auteurs, qui accordent une importance égale aux faits d'actualité. Pour comprendre la fonction de l'image artistique dans les œuvres communes de Kluge/Richter et son rapport critique au thème dégagé pour notre lecture, il faut se pencher quelques instants sur la genèse du livre Nachricht von ruhigen Momenten. Le projet d'un deuxième livre commun est notamment lié à l'édition du journal allemand Die Welt du 5 octobre 2012, la rédaction ayant choisi de confier ce jour-là la responsabilité de la «mise en images » à Gerhard Richter (Georg Baselitz et Ellsworth Kelly l'avaient déjà fait avant lui et le principe s'est répété avec Neo Rauch le 30 octobre 2013, puis avec Cindy Sherman le 8 octobre 2014). Le 4 octobre 2012 à 10 heures, Richter arrive dans les locaux du journal avec une présélection de 116 photos, et après une longue journée de travail (dont on peut suivre le déroulement dans le «Minutenprotokoll» publié sur le site internet de Die Welt $^{32}$ ), le numéro part à l'impression avec 45 photos de l'artiste. À l'incohérence et au hasard des faits divers qui constituent le journal s'oppose une construction très réfléchie de Richter, partant de photographies à caractère plutôt privé, parfois même (en apparence) anodin. Les informations livrées dans les 30 pages journalières ne sont plus alors illustrées par des images d'actualité mais agencées avec des photographies sans lien directement apparent (ou évident) avec les nouvelles rapportées.

Dans Nachricht von ruhigen Momenten, un an plus tard, un texte de Kluge intitulé « Künstlerisches Foto aufgrund eines Missverständnisses ${ }^{33}$ » (littéralement « photo

${ }^{30}$ Ibid., p. 50.

31 « Daß die Waldarbeiter gerade in diesem Moment mit ihrer Säge innehielten und diese Struktur entstand, ist Zufall » (ibid., p. 51).

${ }^{32}$ Tim Ackermann, « Richters "Welt" geht in den Druck. Minutenprotokoll », Die Welt, 4.10.2012, http://www.welt.de/kultur/article109617619/Richters-Welt-geht-in-den-Druck.html.

${ }^{33}$ Kluge, Richter, Nachricht von ruhigen Momenten, p. 87. 
d'art sur fond de malentendu ») thématise la tension entre photographies de presse et photographies d'art, dans un contexte où les unes sont semblablement remplacées par les autres, mais cette fois de manière non délibérée: un photographe d'art reçoit la mission de photographier les combats au Mali pour Le Monde, par erreur, à la place de son cousin, photographe de presse. Il prend le parti de photographier des détails (une fourmi, des palmiers ou la surface de l'eau, par exemple). Au début, ces clichés irritent la rédaction du journal, mais ils finissent par plaire au rédacteur en chef, en particulier celui d'un vieux carreau de marbre griffé et fissuré : « Tatsächlich erlaubte es die thematische Unbestimmtheit der Bilder mit Hilfe einer Bildunterschrift, dem festgehaltenen Ausschnitt der Wirklichkeit auf die Sprünge zu helfen ${ }^{34}$ », littéralement: "L'indétermination thématique des images a permis de manière effective, à l'aide d'une légende, de mettre le fragment de réalité fixé par la photo sur la voie, de le faire avancer en quelque sorte, de le faire rebondir ».

Le projet de Die Welt n'était évidemment pas de transformer un numéro du journal en œuvre d'art, mais, d'une part, de remettre en question le statut de l'image comme illustration et, d'autre part, de permettre stratégiquement à l'image artistique de s'insérer à un endroit inattendu (le cœur battant de l'actualité). L'importance de la présence de l'art là où on ne l'attend pas se traduit dans l'un des plans de l'interview filmée que Richter accorde à Kluge le jour-même, dans les locaux du quotidien Die Welt: après avoir été prononcés, les mots «Kunst an unerwartetem Ort ${ }^{35}$ sont repris comme citation en image fixe, durant quelques secondes ${ }^{36}$.

Ce n'est pas la première fois que Richter organise pareille rencontre entre journal de presse et photographie. En mai 2002, l'artiste réalise 216 clichés de détails de sa peinture Abstraktes Bild (Nr. 648-2) de $1987^{37}$, fragments photographiques qu'il combine deux ans plus tard avec des articles du quotidien allemand Frankfurter Allgemeine Zeitung parus au début de la guerre en Irak, les 20 et 21 mars 2003. Le résultat est publié dans un livre intitulé $\mathrm{War} \mathrm{Cut}^{38}$, " coupure de guerre ». Richter reprend d'ailleurs l'une de ces 216 photos dans le second livre qu'il réalise avec Kluge $^{39}$, renvoyant ainsi lui-même à ce premier projet de montage art/presse et nous invitant à mettre les deux projets en rapport. Une différence saute aux yeux: au niveau de la production, les photos utilisées dans la première expérience sont des fragments d'un tableau abstrait (intitulé d'ailleurs Abstraktes Bild), celles utilisées pour la seconde représentent des fragments de réalité et s'inscrivent donc dans le domaine de la figuration. Les images reprises dans Décembre pourraient avoir un statut intermédiaire. Réalisées à partir de la réalité, et donc figuratives dans leur production, elles prennent pour l'observateur, en raison de leur monotonie, un sens presque abstrait.

34 Ibid.

35 « De l'art là où on ne l'attend pas ».

${ }^{36}$ Kluge, Gerhard Richter: Bildermacher.

37 Voirlesite de l'artiste: www.gerhard-richter.com/en/art/paintings/abstracts/abstracts-19851989-30/ abstract-painting-7633 et www.gerhard-richter.com/en/art/microsites/war-cut-ii.

38 Gerhard Richter, War Cut, Köln, Walther König, 2004.

39 Kluge, Richter, Nachricht von ruhigen Momenten, p. 85. 
On ne peut cependant pas opposer trop frontalement l'image abstraite et l'image figurative. En tant que spécialiste de l'art visuel, Richter sait que l'œil humain tend à effacer cette différence au niveau de la perception. C'est ce qui est mis en évidence par le texte intitulé « Kritik an einem Kriegsausbruch durch Fragmentierung eines Gemäldes » dans Alexander Kluge in Halberstadt ${ }^{40}$. Le sujet en « je » du récit, Bernd Künneke, aperçoit un soldat dans l'un des détails photographiés du projet War Cut; on lui répond que Richter ne pensait pas de manière figurative en réalisant le tableau et que ce qu'il voit est seulement le produit du hasard ${ }^{41}$. Bernd Künneke trouve néanmoins les images « déroutantes » (« unruhestiftend ») et y voit très souvent des éléments figuratifs (un archipel dans les mers du Sud, une tranchée ou la forme des États-Unis par exemple), même s'il décide finalement de taire ses impressions, puisqu'elles sont rejetées par ses interlocuteurs et qu'elles contredisent la catégorie même dans laquelle le titre place le tableau Abstraktes Bild. Mais la remarque de Gerhard Richter dans l'interview de 2012 sur ses peintures dites «abstraites » renforce l'hypothèse d'une indétermination fondamentale du genre de l'expression: il défend l'idée que ses tableaux ne sont pas purement abstraits, qu'on y voit toujours un sens, tout simplement parce que nous sommes toujours à la recherche d'un sens ${ }^{42}$. Le sens se constitue ici par la réception, par celui qui regarde, et (en partie du moins) par hasard.

Comment donc un numéro de journal contenant 45 photos de Richter a-t-il pu déboucher un an plus tard sur un livre commun? La veille de la sortie du journal Die Welt, en octobre 2012, Kluge était avec Richter dans les bâtiments de la rédaction (c'est d'ailleurs dans ce contexte qu'il l'a interviewé) et annonçait déjà que la sélection de photos lui avait inspiré une trentaine de petits textes, pour un nouveau projet de livre à deux. Un de ces textes figure déjà dans le journal du 5 octobre 2012, pratiquement à l'identique. Il accompagne la photo d'un chien couché au soleil et est signé «Alexander Kluge ». C'est aussi le seul endroit du journal où texte et image ont un lien directement évident, où l'on voit que le texte décrit l'image ou que l'image illustre le texte, dans un rapport assez traditionnel. Dans le livre, près d'un an plus tard, on retrouve le texte et l'image, mais sans qu'ils soient juxtaposés ${ }^{43}$.

On peut deviner ce que Kluge trouvait de stimulant dans l'expérience du numéro spécial et qui a éveillé son intérêt. Le titre Nachricht von ruhigen Momenten semble d'ailleurs traduire l'effet supposé des images de Richter intégrées au journal d'octobre 2012. Kluge le dit lui-même dans l'interview de Richter, à propos de la photo du chien couché au soleil: « Es kommt durch die Bilder eine RUHE hinein » (littéralement «à travers les photos il y un CALME qui s'installe »). Une collection de «moments calmes» donc, à l'opposé des actualités de la presse (on retrouve « Nachricht» au singulier dans le titre du livre, qu'on peut opposer aux « (Presse-)

40 Alexander Kluge, « Kritik an einem Kriegsausbruch durch Fragmentierung eines Gemäldes », in Thomas Combrink, Alexander Kluge, Alexander Kluge in Halberstadt, Halberstadt, Gleimhaus, 2013, p. 35 .

41 « Mein Eindruck beruhe auf der Deutung eines Zufalls » (ibid.).

${ }^{42}$ Cf. Kluge, Gerhard Richter: Bildermacher: « [...] meine Bilder sind nicht abstrakt - wir sehen darin einen Sinn, weil wir immer einen Sinn suchen, wir wollen immer deuten ».

${ }^{43}$ Kluge, Richter, Nachricht von ruhigen Momenten, p. 13 (pour la photo) et p. 33 (pour le texte). 
Nachrichten »). Au mouvement continuel de la presse quotidienne s'oppose la volonté de l'artiste de calmer le jeu, de ralentir le temps en le fixant dans quelques instants calmes, par l'image et par les mots. Un contraste, une tension que souligne la maison d'édition Suhrkamp dans la présentation du livre: « Des images de moments calmes à une époque mouvementée [...] le for privé au lieu de l'histoire du monde ${ }^{44} \gg$. Voilà bien une autre réponse de l'art à l'emportance du temps: contrebalancer la fureur de Chronos par des moments de suspens, déranger sa trajectoire linéaire en proposant de multiples autres voies (notamment pour l'interprétation des faits rapportés), déstabiliser les idées toutes faites en les complexifiant. Comme dans Décembre, on retrouve plusieurs faits divers de type journalistique (des accidents, catastrophes naturelles, anecdotes, etc.). Mais ce que Kluge problématise relève bien de la tension, qu'elle soit inhérente à ces histoires (en raison notamment du hasard qui les détermine), ou créée par les montages inédits dans lesquels sont pris textes et images. Ou encore qu'elle s'installe entre plusieurs images.

Dans l'interview de Richter, Kluge évoque lui-même la tension entre la réalité et les flous de la peinture ${ }^{45}$. Le flou est très largement utilisé dans le numéro de Die Welt mis en images par Richter (12 photographies sur 45 sont privées de netteté). Certaines photographies apparaissent floues dans l'édition spéciale de Die Welt alors qu'elles sont nettes dans le livre - on peut en déduire qu'elles ont été floutées délibérément pour le journal, probablement dans l'idée de relativiser quelque peu leur caractère privé, de leur donner une valeur plus générale et d'éviter l'indiscrétion.

Constitutive du livre et de sa genèse, la tension entre presse et art se voit directement thématisée dans certains textes de Kluge. Deux idées principales s'y expriment: (1) l'image quotidienne de la réalité de notre monde est constituée/ formatée par les nouvelles des journaux, et non pas par les faits en tant que tels ${ }^{46}$; (2) l'actualité constitue notre rythme collectif $^{47}$. Face à cette cadence effrénée des nouvelles, l'art permet un possible contre-pied: «Les productions poétiques constituent un contraste avec ce changement quotidien. Dans les peintures, dans les récits des nouvelles et des romans, le temps s'arrête autour de nous ${ }^{48}$ ».

Kluge et Richter partagent l'idée que l'expérience humaine est liée à une fixation du temps par les mots (dans le récit oral ou écrit) ou par l'image (dans la photographie, la peinture ou le film). Kluge écrit « Man erlebt, was erzählt wird ${ }^{49}$ » - on vit ce qui est raconté. Le tout premier texte du livre, qui par sa position acquiert une importance particulière, met en scène un citadin ayant passé un jour de congé à

44 « Bilder von ruhigen Momenten in unruhigen Zeiten, [...] Privates statt Welthistorisches ». http:// www.suhrkamp.de/buecher/nachricht_von_ruhigen_momenten-alexander_kluge_22477.html.

45 «Wenn es eine Spannung kriegt, ist es interessant» (Kluge, Gerhard Richter: Bildermacher).

46 «Aus solchen NACHRICHTEN-WERTEN, nicht aus den Tatsachen selbst, ist das tägliche Bild der Wirklichkeit unserer Welt zusammengesetzt » (Kluge, Richter, Nachricht von ruhigen Momenten, p. 83); «Zuständig für die Kenntnis dessen, was in der Wirklichkeit geschieht, bleiben die Zeitungen mit ihren Bildern » (ibid., p. 109).

47 «Ohne solche Nachrichten aber wäre das, was die Sinne in der Nähe erfahren, ohne kollektiven Rhythmus. » (ibid.).

48 « Poetische Produkte bilden zu diesem täglichen Wechsel einen Gegensatz. In gemalten Bildern, in den Erzählungen der Novellen und Romane steht die Zeit draußen still » (ibid., p. 83).

49 Ibid., p. 127. 
la campagne avec ses deux enfants et tentant de fixer les impressions de ce jour férié qui s'envole (« die Eindrücke des dahinfliegenden Feiertages $\left.{ }^{50} »\right)$. Il pense pouvoir le faire en les racontant à quelqu'un; finalement ce ne sont pas des mots qui lui viennent naturellement mais des images (il dit qu'il essaiera de trouver plus tard les mots adéquats). Il se demande pourquoi chercher ainsi à retenir des images et se convainc de leur valeur unique, qui ne se répète pas («Es ist ein "Wert", der sich nicht wiederholt »). Il en conclut que la fonction principale de l'image est de fixer l'éphémère et se souvient alors d'une image vue deux jours auparavant: des fleurs blanches flottant au vent dans une cour en béton, « des fleurs en un lieu nonpoétique » («Blüten am unpoetischen Ort »), à un endroit inattendu. Probablement un hasard, une convergence hasardeuse d'événements qu'il ne croisera pas deux fois dans sa vie («Auch dies ein Zufall von Ereignissen, von denen er nicht annahm, dass er ihnen zweimal im Leben begegnen würde »).

Comment résister à l'emportance du temps? En privilégiant l'instant très bref où tout peut encore arriver, le photographe saisit par son art - et « pétrifie » ou « gèle » en quelque sorte - un moment de tension entre plusieurs événements susceptibles de survenir. C'est ce qui s'appelle « dompter le hasard », pour reprendre la formule que Kluge emprunte lui-même à Benjamin. Saisir un tel moment de tension permet de réintroduire des indéterminations là où le temps (Chronos) travaille sans cesse à les réduire. L'écrivain peut obtenir des effets semblables en racontant autrement une histoire bien connue, en ajoutant des détails (fictifs ou réels) capables de tout faire basculer. L'actualité constitue certainement l'un des domaines d'application les plus intéressants de telles stratégies. Alexander Kluge et Gerhard Richter nous ont aidés à saisir ce fait essentiel: l'indétermination des œuvres (photographies artistiques, ici) permet de donner de la profondeur au moment de la réception. De « réintroduire du calme », de « postposer » les analyses trop directes et univoques, de décaler nos réactions. La tension qu'autorise l'image ouvre un espace dans lequel nous pouvons transformer nos représentations du monde.

${ }^{50}$ Ibid., p. 10. 